\section{Maintaining NET balance}

\section{By Chris Cain, Staff Writer}

In cystic fibrosis, the rationale for antagonizing CXC chemokine receptor 2 has been to minimize the recruitment of immune cells to the inflamed lung by the receptor's chemokine ligands. Now, a team at the University of Tuebingen has shown that antagonists of the receptor also can stop the formation of neutrophil extracellular traps, which contribute to the buildup of DNA and protein that impairs lung function in CF patients. ${ }^{1}$

The mechanistic link uncovered here could mean that inhibitors of CXC chemokine receptor 2 (CXCR2; IL8RB) also have therapeutic potential for other inflammatory diseases in which neutrophil extracellular traps (NETs) play a role, such as systemic lupus erythematosus (SLE), vasculitis and deep vein thrombosis. ${ }^{2,3}$

NETs are secreted by neutrophils to ensnare and destroy pathogens, and they consist of neutrophil DNA, chromatin proteins and antimicrobial peptides. ${ }^{4}$ During chronic inflammation, neutrophil activation can lead to excess NET

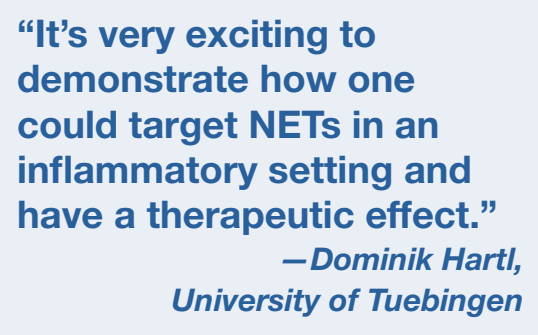

Data were published in Nature Medicine.

"The view of many people is that NETs act as a double-edged swordit's unclear whether their antibacterial benefit outweighs their negative effects in inflammatory disease," Hartl told SciBX. "It's very exciting to demonstrate how one could target NETs in an inflammatory setting and have a therapeutic effect."

\section{NET's present value}

Antagonizing CXCR2 is not a new idea for treating diseases stemming from pulmonary inflammation. At least six companies have CXCR2 antagonists in development stages ranging from preclinical to Phase II to treat $\mathrm{CF}$, asthma, chronic obstructive pulmonary disease (COPD) and respiratory distress syndrome. The two most advanced compounds are Merck \& Co. Inc.'s SCH527123, which is in Phase II clinical trials to treat asthma and COPD, and GlaxoSmithKline plc's SB-656933, which is in a Phase II clinical trial to treat CF.

Although blocking CXCR2 is known to reduce inflammation by impairing neutrophil recruitment, the new findings suggest it also may minimize the symptoms caused by neutrophils already in the airway.

In an e-mail to $S c i B X$, Richard Chapman, distinguished research fellow of respiratory immunology, and Daniel Lundell, senior director, both at Merck Research Laboratories, noted that "blockade of chemotaxis was thought to be the major mechanism by which CXCR2 inhibitors would work to treat airway inflammation."

The current paper "shows that CXCR2 (not CXCR1) is also involved in 'NETosis'. This finding adds an important new process controlled formation and can cause disease.

In SLE, for example, NETs may contribute to the production of antibodies against chromatin and neutrophil proteins, which themselves help drive the disease's autoimmune assault. ${ }^{2}$

For several years, Dominik Hartl, professor of pediatric infectious diseases and immunology at the University of Tuebingen, has been looking for a receptor on neutrophils responsible for mediating NET formation. Now, through a series of cell culture studies, his team thinks it has the answer-CXCR2.

To determine whether the mechanism is relevant to disease, the team collected sputum from CF patients and found that it induced NET formation compared with sputum from healthy patients. An antagonist of CXCR2 significantly decreased NET formation compared with vehicle control $(p<0.05)$.

The team also examined NET formation in a mouse model of CF. Intra-airway administration of a CXCR2 antagonist significantly decreased NET formation and increased lung function compared with administration of vehicle control.

Importantly, blocking NET formation in the airway did not increase bacterial proliferation when the animals were infected with Pseudomonas aeruginosa, a common opportunistic pathogen in CF. These findings suggest that blocking NETs could be done without compromising the immune system. by CXCR2," they wrote.

According to Lundell and Chapman, "current treatment for CF includes antibacterials, antimucolytic agents and bronchodilators. A CXCR2 inhibitor would work by an entirely new mechanism; it may work with these treatments to improve overall therapy."

With a mechanism for inhibiting NETs in hand, it may be possible to expand the use of CXCR2 antagonists to additional inflammatory diseases. But it could be a double-edged sword. Although coagulation elicited by NETs may help protect organisms from systemic infections, one consequence is that inappropriate NET formation could cause thrombosis.

This year, two other research groups have shown that NETs induce coagulation and thrombosis.,

In a baboon model of induced deep vein thrombosis, researchers at Harvard University showed that NETs were strongly associated with thrombi.

Meanwhile, Bernd Engelmann, professor of clinical chemistry at the Ludwig Maximilian University of Munich, led another group showing a role for NETs in coagulation.

"Any disease linked to strong activation of neutrophils would be of interest to consider treating by inhibiting NETs, for example rheumatoid and other obstructive lung diseases in addition to cystic fibrosis," he told 


\section{ANALYSIS}

\section{TARGETS \& MECHANISMS}

SciBX. "You must be careful to maintain an appropriate level of neutrophils, and in diseases where you have high numbers of neutrophils, antagonizing CXCR2 may work well."

Engelmann did note that CXCR2 plays many roles in vivo, and its antagonism may have widespread effects on immunity.

Still, despite the target's myriad roles, antagonizing CXCR2 could yield better results in NET-related diseases than directly targeting NETs with agents such as DNase, which have been shown to be effective in CF. The reason is that the combination of DNA and protein that comprises NETs may be resistant to DNases, at least in SLE.

Roche's Genentech Inc. unit markets Pulmozyme, an inhaled recombinant DNase, to treat $\mathrm{CF}$.

"Breaking up NETs with DNase may work as a therapeutic, but it can only act on existing NETs, and it is unclear if the broken-down NETs would cause residual problems," said Hartl. "It would be better to act to prevent NET formation in the first place."

Engelmann suggested that nucleosomes, which are only found outside of the cell when they are components of NETs, may provide another target for inflammatory diseases and thrombosis. His team demonstrated that antibodies to nucleosomes decreased blood clotting in a mouse model of systemic bacterial infection compared with control
IgG and that they also prevented arterial thrombosis, the main inducer of myocardial infarction and stroke.

Neither Hartl nor Engelmann have filed for patents on their findings on NET inhibition.

Cain, C. SciBX 3(37); doi:10.1038/scibx.2010.1111

Published online Sept. 23, 2010

\section{REFERENCES}

1. Marcos, V. et al. Nat. Med.; published online Sept. 5, 2010; doi: $10.1038 / \mathrm{nm} .2209$

Contact: Dominik Hartl, University of Tuebingen, Tuebingen, Germany e-mail: dominik.hart|@med.uni-tuebingen.de

2. Hakkim, A. et al. Proc. Natl. Acad. Sci. USA 107, 9813-9818 (2010)

3. Kessenbrock, K. et al. Nat. Med. 15, 623-625 (2009)

4. Brinkmann, V. et al. Science 303, 1532-1535 (2004)

5. Fuchs, T.A. et al. Proc. Natl. Acad. Sci. USA 107, 15880-15885 (2010)

6. Massberg, S. et al. Nat. Med. 16, 887-896 (2010)

COMPANIES AND INSTITUTIONS MENTIONED

Genentech Inc., South San Francisco, Calif. GlaxoSmithKline plc (LSE:GSK; NYSE:GSK), London, U.K. Harvard University, Boston, Mass.

Ludwig Maximilian University of Munich, Munich, Germany

Merck \& Co. Inc., Whitehouse Station, N.J.

Roche (SIX:ROG; OTCQX:RHHBY), Basel, Switzerland

University of Tuebingen, Tuebingen, Germany 